\title{
Growing the International Reach and Accessibility of IJSPP
}

How might we grow the international nature of the International Journal of Sports Physiology and Performance (IJSPP) and other sport-science-related collaborations and activities? The 16-year history of IJSPP since inception has seen tremendous growth, but the essential elements, including a US-based publisher (Human Kinetics), an English-language format, both the editorial team and editorial board predominantly from English-speaking countries, and correspondingly a large proportion of published articles from the same countries. In this era of diversity and inclusion, the issue of how to broaden the focus and reach to other countries with a different culture, language(s), and socioeconomic status has never been more prominent. Fortunately, the basic nature of exercise and sport is universal, and this provides the common "language" and interest for all readers and contributors.

The research of several of the world's leading sporting nations including Russia, China, Japan, and South Korea (all top 8 nations at the 2016 Summer Olympic Games) does not feature very regularly in IJSPP. This is a historical legacy and probably consistent with other sport and exercise science journals that publish in English. The researchers from these countries may not have enjoyed the publication opportunities of their English-speaking counterparts, but in the same light, the English-speaking nations may have missed out on important research initiatives, innovations, and outcomes originating from these sporting giants.

Muslims make up about a quarter of the world's population, and Islam is the second largest religion after Christianity. One of the religious rituals of healthy adult Muslims is fasting from sunrise to sunset during Ramadan. ${ }^{1}$ This is one exception to the dearth of published articles from the Muslim world-Ramadan and its effects on exercise and sporting performance has generated many articles often involving international collaborations. Researchers should look for similar collaborative opportunities across national, religious, cultural, and/or regional lines.

Apart from the obvious challenges for non-English-nativespeaking researchers is the lack of access to language translating services and their cost, often in combination with a weaker local currency. This is one opportunity for native English-speaking researchers to provide guidance and expertise to a collaborative project (manuscript). Of course, the barriers to publication are likely additive, and it is easy to imagine the challenges faced by female researchers in low- and middle-income countries. The gender issues around opportunities in sport; science, technology, engineering, and mathematics; and academia are well known, but effective solutions have been slow to emerge. Increased awareness and both individual and institutional leadership can be implemented immediately. Gender issues continue to be a priority as there is much work to be done in exercise and sport science to improve both the quality and the scope of the research itself, ${ }^{2}$ but also to ensure equity in opportunities for all researchers.

The COVID-19 pandemic has changed the nature of collaborations, with videoconferencing now a primary and acceptable means of delivering lectures, conducting conferences, and for a wide variety of formal and informal communications. Several research social networking and exchange platforms have emerged and are widely used to share papers, pose and answer questions, and find collaborators. Responding to requests for papers is a quick and easy process and very much appreciated by students and emerging researchers.

It is best to develop local, national, and international collaborations at the conceptual or project formulation stage, rather than later during data collection or manuscript preparation. However, collaboration on projects and manuscripts should proceed on a case-bycase basis. Most contemporary guidelines for authorship require a substantial contribution from coauthors across the life span of a research project, not just the manuscript itself. Often opportunities for collaboration come mid- to late career once a researcher's profile and publication track record has been well established. However, with digital accessibility so widespread nowadays, there are fewer limits on contacting any other researcher. Encouraging doctoral students or early-career researchers to contact and network with each other is also a useful strategy.

A substantial amount has been written about pay-to-publish and pay-to-read journals in the online publication world. IJSPP via its publisher Human Kinetics requires a modest submission fee, but there are no page charges for authors. Some publishers and journals offer a discount or waiver by arrangement for researchers (and student researchers) in developing countries or regions. The challenges and barriers of paying moderate to high article-processing charges remain an issue for a very large number of researchers. ${ }^{3}$ Researchers should lobby their institutions and professional societies to identify and develop means of offsetting publication costs. Others can debate the relative merits of different publication models, but in the spirit of cooperation, authors with access to funding could support less-fortunate international colleagues.

There are so many ways now for sport scientist, researchers, academics, and readers to interact. Online forums, blogs, and social media also offer many opportunities to connect near and far. To that end, IJSPP is encouraging authors and readers to communicate more easily with posting of Twitter handles on IJSPP manuscripts. ${ }^{4}$ Twitter can be a very useful 2-way mechanism of pushing out research publications, and connecting with researchers and research groups seeking collaborative opportunities. Creating an international contact or collaboration can start today and just might yield a small project, a manuscript, and occasionally a more enduring partnership.

\section{David B. Pyne, Editor Emeritus, IJSPP}

\section{References}

1. Moghadam MT, Taati B, Ardakani SMP, Ramadan K. Fasting during the COVID-19 pandemic; observance of health, nutrition and exercise criteria for improving the immune system. Front Nutr. 2021;7: e570235.

2. Nimphius S. Exercise and sport science failing by design in understanding female athletes. Int J Sports Physiol Perform. 
2019;14(9):1157-1158. PubMed ID: 31553942 doi:10.1123/ IJSPP.2019-0703

3. Jain VK, Iyengar KP, Vaishya R. Article processing charge may be a barrier to publishing. J Clin Orthop Trauma. 2021;14:14-16. PubMed ID: 33680812 doi:10.1016/j.jcot.2020.10.039
4. De Koning JJ, van Erp T, Lamberts RP, Cheung S, Noordhof D. The IJSPP Twitter account: our secondary step to narrow the gap between sport science and sport practice. Int J Sports Physiol Perform. 2020;16(2):161-162. PubMed ID: 33421959 doi:10.1123/IJSPP. 2020-0956 